\title{
Attainability of the best Sobolev constant in a ball
}

\author{
Norisuke Ioku* \\ Graduate School of Science and Engineering, Ehime University \\ Matsuyama, Ehime 790-8577, Japan
}

\begin{abstract}
The best constant of the Sobolev inequality in the whole space is attained by the Aubin-Talenti function; however, this does not happen in bounded domains because the break in dilation invariance. In this paper, we investigate a new scale invariant form of the Sobolev inequality in a ball and show that its best constant is attained by functions of the Aubin-Talenti type. Generalization to the Caffarelli-Kohn-Nirenberg inequality in a ball is also discussed.
\end{abstract}

Keywords: Sobolev inequality, Caffarelli-Kohn-Nirenberg inequality, Best constant, Attainability, Scale invariance

2010 MSC: Primary; 35K55, Secondly; 35A01, 46E30

\section{Introduction and Results}

The Sobolev inequality states that, if $n \geq 2$ and $1 \leq p<n$, then

$$
S_{n, p}\|u\|_{L^{p^{*}}} \leq\|\nabla u\|_{L^{p}}
$$

for every $u \in W^{1, p}\left(\mathbb{R}^{n}\right)$, where $p^{*}$ is the Sobolev conjugate number defined by $p^{*}=n p /(n-p)$ and $S_{n, p}$ is the best constant for the inequality (1.1) given by

$$
\begin{cases}S_{n, p}=\sqrt{\pi} n^{\frac{1}{p}}\left(\frac{n-p}{p-1}\right)^{\frac{p-1}{p}}\left[\frac{\Gamma\left(\frac{n}{p}\right) \Gamma\left(n+1-\frac{n}{p}\right)}{\Gamma(n) \Gamma\left(1+\frac{n}{2}\right)}\right]^{\frac{1}{n}} & \text { for } 1<p<n, \\ S_{n, 1}=\sqrt{\pi} \frac{n}{\left[\Gamma\left(1+\frac{n}{2}\right)\right]^{\frac{1}{n}}} & \text { for } p=1,\end{cases}
$$

where $\Gamma(\cdot)$ is the gamma function. The inequality (1.1) with the best constant was obtained by Federer-Fleming [10] and Maz'ya [16] for $p=1$ and by Aubin [3] and

*e-mail address: ioku@ehime-u.ac.jp 
Talenti [20] for $1<p<n$. Furthermore, if $1<p<n$, the best constant is attained by the two parameter family

$$
U(x)=\left(a+b|x|^{\frac{p}{p-1}}\right)^{1-\frac{n}{p}}, \quad a, b>0
$$

and its translation. If we replace $\mathbb{R}^{n}$ in $\Omega \subset \mathbb{R}^{n}$, the Sobolev inequality still holds for $u \in W_{0}^{1, p}(\Omega)$ with the same best constant; however, it is not attained in $W_{0}^{1, p}(\Omega)$ because the dilation invariance under

$$
u_{\mu}(x)=\mu^{\frac{n-p}{p}} u(\mu x), \quad \mu>0,
$$

breaks (see [14, p.44] or [23, Proposition 1.43]).

On the other hand, in the critical case of $p=n$, the Sobolev inequality of the form (1.1) is no longer true because $S_{n, p} \rightarrow 0$ as $p \rightarrow n$, and $W_{0}^{1, n}(\Omega)$ is not embedded into $L^{\infty}(\Omega)$. Alvino [2] considered the critical case and obtained

$$
\sqrt{\pi} \frac{n^{\frac{1}{n}}}{\Gamma\left(1+\frac{n}{2}\right)^{\frac{1}{n}}} \sup _{x \in B_{R}} \frac{\left|u^{*}(x)\right|}{\left(\log \left(\frac{R}{|x|}\right)\right)^{\frac{n-1}{n}}} \leq\|\nabla u\|_{L^{n}\left(B_{R}\right)} \quad \text { for all } u \in W_{0}^{1, n}\left(B_{R}\right)
$$

where $B_{R}$ is the ball centered at the origin with radius $R>0$ and $u^{*}$ denotes the Schwarz symmetrization of $u \in W_{0}^{1, n}\left(B_{R}\right)$. For the definition of $u^{*}$, see the end of Section 1 of this paper or [14, Section 1.3]. Alvino's inequality (1.4) is known to be the critical case of Sobolev embeddings because this inequality implies the optimal embedding of $W_{0}^{1, n}\left(B_{R}\right)$ into Orlicz spaces (see [7, Example 1]). Several equivalent forms of (1.4), especially the relationship between (1.4) and Moser-Trudinger inequalities, are discussed by Cassani-Sani-Tarsi [6].

One of the main difference between the Alvino inequality (1.4) and the Sobolev inequality (1.1) is the scale invariance structure, that is, the critical case (1.4) is not invariant under the dilation $x \mapsto \lambda x$ but is invariant under

$$
u_{\lambda}(x)=\lambda^{-\frac{n-1}{n}} u\left(\left(\frac{|x|}{R}\right)^{\lambda-1} x\right), \quad \lambda>0 .
$$

This scaling was firstly found by Adimurthi-do Ó-Tintarev [1], and they pointed out that Moser functions are invariant under (1.5). Cassani-Ruf-Tarsi [5] proved that Moser functions are the minimizers of the minimizing problem associated with (1.4) by focusing its invariance under (1.5). Costa-Tintarev [8] applied the scaling (1.5) to analyze the concentration profiles of the Trudinger-Moser functional.

It is well known that the inequalities (1.1) and (1.4) with the best constant have vast applications and generalizations in geometry, physics, and functional analysis. Even though (1.1) and (1.4) are widely studied, several questions still arise naturally from the view-point of attainability of the best constant and the scale invariance property. Does the Sobolev inequality in $W_{0}^{1, p}\left(B_{R}\right)$ have a scale invariant form? Can we obtain Alvino's inequality (1.4) from the Sobolev type inequalities by directly 
limiting the procedure $p \rightarrow n$ ? Is there any relationship between the two scalings: the dilation (1.3) for $W^{1, p}\left(\mathbb{R}^{n}\right)$ and the scaling (1.5) for $W_{0}^{1, n}\left(B_{R}\right)$ ?

In this paper, we give a positive answer for these questions by discovering the scale invariant form of a Sobolev type inequality in $W_{0}^{1, p}\left(B_{R}\right)$, which recovers the attainability of the best constant and implies Alvino's inequality by taking $p \rightarrow n$.

To state our result, we introduce the $q$-logarithmic function and $q$-exponential function as follows:

$$
\begin{aligned}
\log _{q} r & :=\frac{r^{1-q}-1}{1-q}, \\
\exp _{q}(r) & :=[1+(1-q) r]^{\frac{1}{1-q}},
\end{aligned}
$$

for $q>0, q \neq 1$ and $r>0$. It is easy to verify that

$$
\lim _{q \rightarrow 1} \log _{q} r=\log r, \quad \lim _{q \rightarrow 1} \exp _{q} r=e^{r} \quad \text { for all } r>0 .
$$

These modified logarithmic, exponential functions were originally introduced by Tsallis [21] to study nonextensive statistics. See [22, Section 3] or [18, Section 2 and Appendix A] for more details on $q$-logarithmic, exponential functions.

The first result is an improved Sobolev inequality for radially symmetric functions.

Theorem 1.1 (Sobolev type inequality). Let $n \in \mathbb{N}, n \geq 2,1<p<n$, and $\frac{1}{p^{*}}=\frac{1}{p}-\frac{1}{n}$. Then for any radially symmetric function $u \in W_{0}^{1, p}\left(B_{R}\right)$ the following holds:

$$
S_{n, p}\left(\frac{n-p}{p-1}\right)^{\frac{1}{n}-1}\left(\int_{B_{R}} \frac{|u(x)|^{p^{*}}}{\left[\log _{\frac{n-1}{p-1}} \frac{R}{|x|}\right]^{\frac{p(n-1)}{n-p}}} d x\right)^{\frac{1}{p^{*}}} \leq\|\nabla u\|_{L^{p}\left(B_{R}\right)} .
$$

The left-hand-side constant is optimal and attained by

$$
U_{R}(x)=\left[a+b\left\{\frac{1}{|x|^{\frac{n-p}{p-1}}}-\frac{1}{R^{\frac{n-p}{p-1}}}\right\}^{-\frac{p}{n-p}}\right]^{1-\frac{n}{p}}
$$

where $a, b>0$. Furthermore, the inequality (1.7) is invariant under the following nonlinear scaling

$$
\left\{\begin{array}{l}
u_{\lambda}(x):=\lambda^{-\frac{p-1}{p}} u\left(x_{\lambda}\right) \\
x_{\lambda}:=\left[\lambda|x|^{-\frac{n-p}{p-1}}+(1-\lambda) R^{-\frac{n-p}{p-1}}\right]^{-\frac{p-1}{n-p}} \frac{x}{|x|} .
\end{array}\right.
$$


Remark 1.1. The inequality (1.7) yields the classical Sobolev inequality in a bounded domain $\Omega$ and non-attainability of the best constant by symmetrization techniques as follows. Let $u \in W_{0}^{1, p}(\Omega)$ and $u^{*}$ be its Schwarz symmetrization, where $B_{R}$ is the ball centered at the origin having the same measure as $\Omega$. Since

$$
\frac{n-p}{p-1} \log _{\frac{n-1}{p-1}} \frac{R}{|x|}=1-\left(\frac{|x|}{R}\right)^{\frac{n-p}{p-1}}<1
$$

for all $x \in B_{R}$, the inequality (1.7) to $u^{*} \in W_{0}^{1, p}\left(B_{R}\right)$ together with the Pólya-Szegö inequality shows that

$$
\begin{aligned}
S_{n, p}\|u\|_{L^{p^{*}}(\Omega)}=S_{n, p}\left\|u^{*}\right\|_{L^{p^{*}\left(B_{R}\right)}} & <S_{n, p}\left(\int_{B_{R}} \frac{\left|u^{*}(x)\right|^{p^{*}}}{\left[\frac{n-p}{p-1} \log _{\frac{n-1}{p-1}} \frac{R}{|x|}\right]^{\frac{p(n-1)}{n-p}}} d x\right)^{\frac{1}{p^{*}}} \\
& \leq\left\|\nabla u^{*}\right\|_{L^{p}\left(B_{R}\right)} \leq\|\nabla u\|_{L^{p}(\Omega)}
\end{aligned}
$$

for all $u \in W_{0}^{1, p}(\Omega)$. Moreover, the strict inequality (1.11) directly shows that there is no extremal function for the classical Sobolev inequality in the ball $B_{R}$.

Remark 1.2. Clearly the equality in (1.10) shows that Theorem 1.1 yields the classical Sobolev inequality in $\mathbb{R}^{n}$ by taking $R \rightarrow \infty$. The scaling (1.5) converges to the dilation (1.3) as $R \rightarrow \infty$, that is,

$$
\lambda^{-\frac{p-1}{p}} u\left(x_{\lambda}\right) \rightarrow\left(\lambda^{\left.-\frac{p-1}{n-p}\right)^{\frac{n-p}{p}}} u\left(\lambda^{-\frac{p-1}{n-p}} x\right), \quad R \rightarrow \infty .\right.
$$

Furthermore, the extremal function $U_{R}(x)$ in (1.8) converges pointwise to the AubinTalenti function $U(x)$ in (1.2) as $R \rightarrow \infty$.

Remark 1.3. While the classical Sobolev inequality (1.1) does not imply Alvino's inequality, Theorem 1.1 yields Alvino's inequality by the direct limiting procedure $p \rightarrow n$. Indeed, the explicit value of $S_{n, p}$ gives us that

$$
S_{n, p}\left(\frac{n-p}{p-1}\right)^{-\frac{n-1}{n}} \rightarrow \frac{\sqrt{\pi} n^{\frac{1}{n}}}{\Gamma\left(1+\frac{n}{2}\right)^{\frac{1}{n}}}
$$

as $p \rightarrow n$. This together with $\lim _{p \uparrow n} \log _{\frac{n-1}{p-1}} \frac{R}{|x|}=\log \frac{R}{|x|}$ yields the desired convergence

$$
S_{n, p}\left(\frac{n-p}{p-1}\right)^{-\frac{n-1}{n}}\left(\int_{B_{R}} \frac{\left|u^{*}(x)\right|^{p^{*}}}{\left[\log _{\frac{n-1}{p-1}} \frac{R}{|x|}\right]^{\frac{p(n-1)}{n-p}}} d x\right)^{\frac{1}{p^{*}}} \rightarrow \frac{\sqrt{\pi} n^{\frac{1}{n}}}{\Gamma\left(1+\frac{n}{2}\right)^{\frac{1}{n}}} \sup _{x \in B_{R}} \frac{\left|u^{*}(x)\right|}{\left(\log \frac{R}{|x|}\right)^{\frac{n-1}{n}}}
$$

as $p \rightarrow n$. Furthermore, the scaling (1.9) coincides with the scaling (1.5) if $p \rightarrow n$, since $\lim _{p \rightarrow n} x_{\lambda}=\left(\frac{|x|}{R}\right)^{\lambda-1} x$. 
Theorem 1.1 does not hold for arbitrary (not necessarily radially symmetric) $W_{0}^{1, p}\left(B_{R}\right)$ functions. To prove this, let us assume that (1.7) holds for arbitrary $W_{0}^{1, p}\left(B_{R}\right)$ functions and then derive a contradiction. Let $d(x)$ be the distance function to the boundary defined by $d(x):=R-|x|$. Since there exists $C>0$ such that $d(x) / C \leq \log _{\frac{n-1}{p-1}} \frac{R}{|x|} \leq C d(x)$ for all $x \in B_{R}$, the inequality (1.7) yields the following Hardy-Sobolev inequality with the weight function:

$$
\left(\int_{B_{R}}|u(x)|^{p^{*}} d(x)^{-\frac{p(n-1)}{n-p}} d x\right)^{\frac{1}{p^{*}}} \leq C\|\nabla u\|_{L^{p}\left(B_{R}\right)} .
$$

The necessary condition for the exponent of the weight function is known in the study of Hardy-Sobolev inequalities with weights(see [15, Theorem 19.10 and Remark 19.13 with $\kappa=1, q=0])$. Since $-\frac{p(n-1)}{n-p}$ is not admissible, the inequality (1.12) cannot hold. Nevertheless, we obtain the following Sobolev type inequality for arbitrary $W_{0}^{1, p}\left(B_{R}\right)$ functions by introducing the differential operator $L_{p}$. The radial derivative $\nabla_{r} u(x)$ and the tangential derivative $\nabla_{\mathbb{S}^{n-1}} u(x)$ are defined by

$$
\nabla_{r} u(x):=\left(\frac{x}{|x|} \cdot \nabla u(x)\right) \frac{x}{|x|}, \quad \nabla_{\mathbb{S}^{n-1}} u(x):=\nabla u(x)-\nabla_{r} u(x) .
$$

We define $L_{p}$ as follows:

$$
L_{p} u:=\nabla_{\mathbb{S}^{n-1}} u(x) /\left[\frac{n-p}{p-1} \log _{\frac{n-1}{p-1}} \frac{R}{|x|}\right]+\nabla_{r} u(x) .
$$

Clearly the following theorem includes Theorem 1.1, since $L_{p} u=\nabla_{r} u=\nabla u$ if $u$ is radially symmetric.

Theorem 1.2. Let $n \in \mathbb{N}, n \geq 2,1<p<n$. It follows that

$$
S_{n, p}\left(\frac{n-p}{p-1}\right)^{-\frac{n-1}{n}}\left(\int_{B_{R}} \frac{|u(x)|^{p^{*}}}{\left[\log _{\frac{n-1}{p-1}} \frac{R}{|x|}\right]^{\frac{p(n-1)}{n-p}}} d x\right)^{\frac{1}{p^{*}}} \leq\left(\int_{B_{R}}\left|L_{p} u(x)\right|^{p} d x\right)^{\frac{1}{p}}
$$

for all $u \in C_{0}^{\infty}\left(B_{R}\right)$. The constant in the left hand side is optimal and attained by $U_{R}(x)$ defined in (1.8). Furthermore, the inequality (1.14) is invariant under the scaling (1.9).

One can further generalize (1.2) to inequalities of the Caffarelli-Kohn-Nirenberg type, which are weighted versions of Sobolev inequalities (See [4, 11]). The CaffarelliKohn-Nirenberg inequality is stated as follows. For any $1<p<n, \theta>1,0 \leq$ $\frac{1}{\theta}-\frac{1}{\sigma} \leq \frac{1}{n}$ it follows that for all $u \in C_{0}^{\infty}\left(\mathbb{R}^{n}\right)$, we have

$$
S\left(\int_{\mathbb{R}^{n}}|x|^{\frac{n \sigma}{p^{*}}}|u(x)|^{\sigma} \frac{d x}{|x|^{n}}\right)^{\frac{1}{\sigma}} \leq\left(\int_{\mathbb{R}^{n}}|x|^{\frac{n \theta}{p}}|\nabla u(x)|^{\theta} \frac{d x}{|x|^{n}}\right)^{\frac{1}{\theta}} .
$$


Furthermore, if $\theta=\sigma$, it follows that for all not necessarily radial functions $u \in$ $C_{0}^{\infty}\left(\mathbb{R}^{n}\right)$, we have

$$
\tilde{S} \int_{\mathbb{R}^{n}}|x|^{\frac{n \theta}{p^{*}}}|u(x)|^{\theta} \frac{d x}{|x|^{n}} \leq \int_{\mathbb{R}^{n}}|x|^{\frac{n \theta}{p}}\left|\nabla_{r} u(x)\right|^{\theta} \frac{d x}{|x|^{n}} .
$$

This homogeneous case is an improvement of (1.15) in the sense that $\left|\nabla_{r} u(x)\right| \leq$ $|\nabla u(x)|$ for all $x \in \mathbb{R}^{n}$. Here $S$ and $\tilde{S}$ denote the best constant of (1.15) and (1.16), respectively. The Caffarelli-Kohn-Nirenberg inequality (2.8) coincides with the Sobolev inequality if $\theta=p$ and $\sigma=p^{*}$. The same inequality holds on a bounded domain $\Omega$; however, the dilation invariance $x \mapsto \lambda x$ is broken. Our main result corresponds with the scale invariance form of (1.15) in a ball. Let $1<p<n, \theta>$ $1,0 \leq \frac{1}{\theta}-\frac{1}{\sigma} \leq \frac{1}{n}, \frac{1}{p^{*}}=\frac{1}{p}-\frac{1}{n}$, and

$$
q=1+\frac{n-p}{p} \frac{\theta}{\theta-1}
$$

For the main result, the differential operator $L_{p, \theta}$ is defined by

$$
L_{p, \theta} u:=\nabla_{\mathbb{S}^{n-1}} u(x) /\left[(q-1) \log _{q} \frac{R}{|x|}\right]+\nabla_{r} u(x) .
$$

This generalize $L_{p}$ in Theorem 1.2 since $L_{p, p}=L_{p}$. Our main result is given below as Theorem 1.3.

Theorem 1.3 (Inequality of the Caffarelli-Kohn-Nirenberg type in $B_{R}$ ). Let $1<$ $p<n, \theta>1,0 \leq \frac{1}{\theta}-\frac{1}{\sigma} \leq \frac{1}{n}, \frac{1}{p^{*}}=\frac{1}{p}-\frac{1}{n}$, and $q=1+\frac{n-p}{p} \frac{\theta}{\theta-1}$. Then, it follows that

$$
S\left(\int_{B_{R}} \frac{|x|^{\frac{n \sigma}{p^{*}}}|u(x)|^{\sigma}}{\left[(q-1) \log _{q} \frac{R}{|x|}\right]^{1+\frac{\theta-1}{\theta} \sigma}} \frac{d x}{|x|^{n}}\right)^{\frac{1}{\sigma}} \leq\left(\int_{B_{R}}|x|^{\frac{n \theta}{p}}\left|L_{p, \theta} u\right|^{\theta} \frac{d x}{|x|^{n}}\right)^{\frac{1}{\theta}}
$$

for all $u \in C_{0}^{\infty}\left(B_{R}\right)$ with the same constant $S$ as given in (1.15). If in addition $\theta=\sigma$, it follows

$$
\tilde{S} \int_{B_{R}} \frac{|x|^{\frac{n \theta}{p^{*}}}|u(x)|^{\theta}}{\left[(q-1) \log _{q} \frac{R}{|x|}\right]^{\theta}} \frac{d x}{|x|^{n}} \leq \int_{B_{R}}|x|^{\frac{n \theta}{p}}\left|\nabla_{r} u\right|^{\theta} \frac{d x}{|x|^{n}}
$$

for all $u \in C_{0}^{\infty}\left(B_{R}\right)$ with the same constant $\tilde{S}$ as given in (1.16).

Moreover, the constants $S$ and $\tilde{S}$ are optimal, and (1.19) and (1.20) are invariant under

$$
\left\{\begin{array}{l}
u_{\lambda}(x):=\lambda^{-\frac{\theta-1}{\theta}} u\left(x_{\lambda}\right), \\
x_{\lambda}:=\left[\lambda|x|^{-\frac{n-p}{p} \frac{\theta}{\theta-1}}+(1-\lambda) R^{-\frac{n-p}{p} \frac{\theta}{\theta-1}}\right]^{-\frac{p}{n-p} \frac{\theta-1}{\theta}} \frac{x}{|x|} .
\end{array}\right.
$$


The Sobolev type inequalities (1.14) and (1.7) can be obtained as a direct consequence from (1.19) by taking $\theta=p, \sigma=p^{*}$.

If $\theta=\sigma=p$, the inequality (1.16) is known as Hardy's inequality and is given as follows:

$$
\frac{n-p}{p} \int_{\mathbb{R}^{n}} \frac{|v(x)|^{p}}{|x|^{p}} \leq \int_{\mathbb{R}^{n}}\left|\nabla_{r} v(x)\right|^{p} d x, \quad v \in W^{1, p}\left(\mathbb{R}^{n}\right),
$$

where the constant is the best possible and the inequality is invariant under the dilation $x \mapsto \lambda x$. Theorem 1.3 gives us a corresponding result which has scale invariance in a ball.

Corollary 1.1. Let $1<p<n$. There holds

$$
\left(\frac{p-1}{p}\right)^{p} \int_{B_{R}} \frac{|u(x)|^{p}}{|x|^{p}\left[\log _{\frac{n-1}{p-1}} \frac{R}{|x|}\right]^{p}} d x \leq \int_{B_{R}}\left|\nabla_{r} u(x)\right|^{p} d x, \quad u \in W_{0}^{1, p}\left(B_{R}\right),
$$

where the constant is optimal and the inequality is invariant under the scaling (1.9).

To the best knowledge of the author, the elemental inequality (1.22) is new and natural because (1.22) derives the critical Hardy inequality

$$
\left(\frac{n-1}{n}\right)^{n} \int_{B_{R}} \frac{|u(x)|^{n}}{|x|^{n}\left(\log \frac{R}{|x|}\right)^{n}} d x \leq \int_{B_{R}}\left|\nabla_{r} u(x)\right|^{n} d x, \quad u \in W_{0}^{1, n}\left(B_{R}\right)
$$

by taking $p \rightarrow n$. For more details on Hardy's inequality, see [9, 12, 13, 17, 19] and the references therein.

We now summarize some notations and basic facts used in this paper. Let $B_{R}$ be the ball centered at the origin with radius $R>0$. The surface measure of the unit sphere $\mathbb{S}^{n-1}$ is denoted by $\omega_{n-1}$. The Schwarz symmetrization of $f$ denoted by $f^{*}$ is defined by

$$
f^{*}(x)=\inf \left\{\lambda>0: \mu_{f}(\lambda) \leq \frac{\omega_{n-1}}{n}|x|^{n}\right\},
$$

where $\mu_{f}(\lambda)$ is the distribution function of $f$. The Schwarz symmetrization $f^{*}$ is radially symmetric and non-increasing. For $f \in W^{1, p}\left(\mathbb{R}^{n}\right)$, the Schwarz symmetrization $f^{*}$ belongs to $W^{1, p}\left(\mathbb{R}^{n}\right)$ and the Pólya-Szegö inequality

$$
\left\|\nabla f^{*}\right\|_{L^{p}\left(\mathbb{R}^{n}\right)} \leq\|\nabla f\|_{L^{p}\left(\mathbb{R}^{n}\right)}
$$

holds. For more details on the Schwarz symmetrization, see [14]. The $q$-logarithmic function and $q$-exponential function are defined by

$$
\log _{q} r:=\frac{r^{1-q}-1}{1-q}, \quad \exp _{q}(r):=[1+(1-q) r]^{\frac{1}{1-q}},
$$

for $q>0, q \neq 1$ and $r>0$. It is easy to verify that

$$
\lim _{q \rightarrow 1} \log _{q} r=\log r, \quad \lim _{q \rightarrow 1} \exp _{q} r=e^{r} \quad \text { for all } r>0 .
$$


See [22, Section 3] or [18, Section 2 and Appendix A] for more details on $q$-logarithmic, exponential functions. The radial derivative $\nabla_{r} u(x)$ and the tangential derivative $\nabla_{\mathbb{S}^{n-1}} u(x)$ are defined by

$$
\nabla_{r} u(x):=\left(\frac{x}{|x|} \cdot \nabla u(x)\right) \frac{x}{|x|}, \quad \nabla_{\mathbb{S}^{n-1}} u(x):=\nabla u(x)-\nabla_{r} u(x) .
$$

We define the differential operator $L_{p, \theta}$ as follows:

$$
L_{p, \theta} u:=\nabla_{\mathbb{S}^{n-1}} u(x) /\left[(q-1) \log _{q} \frac{R}{|x|}\right]+\nabla_{r} u(x) .
$$

The function of the Aubin-Talenti type (1.8) and the scaling (1.21) can be written by using the $q$-logarithmic and exponential functions, that is,

$$
\begin{aligned}
U_{R}(x) & =\left[a+b\left\{\frac{1}{|x|^{\frac{n-p}{p-1}}}-\frac{1}{R^{\frac{n-p}{p-1}}}\right\}^{-\frac{p}{n-p}}\right]^{1-\frac{n}{p}} \\
& =\left[a+b R^{\frac{p}{p-1}}\left\{-\frac{n-p}{p-1} \log _{\frac{n-1}{p-1}} \frac{|x|}{R}\right\}^{-\frac{p}{n-p}}\right]^{1-\frac{n}{p}}
\end{aligned}
$$

and

$$
\left\{\begin{array}{l}
u_{\lambda}(x):=\lambda^{-\frac{\theta-1}{\theta}} u\left(x_{\lambda}\right), \\
x_{\lambda}:=\left[\lambda|x|^{-\frac{n-p}{p} \frac{\theta}{\theta-1}}+(1-\lambda) R^{-\frac{n-p}{p} \frac{\theta}{\theta-1}}\right]^{-\frac{p}{n-p} \frac{\theta-1}{\theta}} \frac{x}{|x|}=R \exp _{q}\left[\lambda \log _{q} \frac{|x|}{R}\right] \frac{x}{|x|} .
\end{array}\right.
$$

These expressions by $q$-functions will be used in the proof of Theorem 1.3 .

\section{Proof of Theorems}

Two proofs of Theorem 1.3 will be given. One proof is based on the scale invariance under (1.21), and for the other one, we prove the equivalence between the classical inequalities (1.15)-(1.16) and Theorem 1.3. Both proofs stand on the scaling (1.21), therefore, we start by their formal derivations.

\subsection{Formal derivation of the scaling (1.21)}

Fix $\lambda>0$ and the radially symmetric functions $u, \phi_{\lambda} \in C_{0}^{\infty}\left(B_{R}\right)$. Define

$$
u_{\lambda}(x):=\lambda u\left(\phi_{\lambda}(x)\right),
$$

and consider the condition on $\phi_{\lambda}$ so that

$$
\int_{B_{R}}|x|^{\frac{n}{p} \theta}\left|\nabla u_{\lambda}(x)\right|^{\theta} \frac{d x}{|x|^{n}}=\int_{B_{R}}|x|^{\frac{n}{p} \theta}|\nabla u(x)|^{\theta} \frac{d x}{|x|^{n}} .
$$


Since $u$ and $\phi_{\lambda}$ are radially symmetric, we have

$$
\int_{B_{R}}|x|^{\frac{n}{p} \theta}\left|\nabla u_{\lambda}(x)\right|^{\theta} \frac{d x}{|x|^{n}}=\omega_{n-1} \int_{0}^{R} \lambda^{\theta} r^{\frac{n}{p} \theta-1}\left|\frac{d}{d r} u_{\lambda}\left(\phi_{\lambda}(r)\right)\right|^{\theta} d r .
$$

Changing the variable $s=\phi_{\lambda}(r)$, we have

$$
\begin{aligned}
\int_{0}^{R} \lambda^{\theta} r^{\frac{n}{p} \theta-1}\left|\frac{d}{d r} u_{\lambda}\left(\phi_{\lambda}(r)\right)\right|^{\theta} d r & =\int_{0}^{R} \lambda^{\theta} r^{\frac{n}{p} \theta-1}\left|s^{\prime} u^{\prime}(s)\right|^{\theta} \frac{1}{s^{\prime}} d s \\
& =\int_{0}^{R} \lambda^{\theta} r^{\frac{n}{p} \theta-1} s^{\prime \theta-1}\left|u^{\prime}(s)\right|^{\theta} d s .
\end{aligned}
$$

Therefore, if $s=\phi_{\lambda}(r)$ satisfies the ordinary differential equation

$$
\lambda^{\theta} r^{\frac{n}{p} \theta-1} \phi_{\lambda}^{\prime \theta-1}=\phi_{\lambda}^{\frac{n}{p} \theta-1}
$$

we obtain the desired invariance. Here (2.1) is solvable by separation of variables and its solution under the boundary condition $\phi_{\lambda}(R)=R$ is

$$
\phi_{\lambda}(r)=\left(\lambda^{-\frac{\theta}{\theta-1}} r^{-\frac{n-p}{p} \frac{\theta}{\theta-1}}+\left(1-\lambda^{-\frac{\theta}{\theta-1}}\right) R^{-\frac{n-p}{p} \frac{\theta}{\theta-1}}\right)^{-\frac{p}{n-p} \frac{\theta-1}{\theta}} .
$$

Taking $\lambda \mapsto \lambda^{-\frac{\theta-1}{\theta}}$, we obtain the scaling (1.21).

\subsection{Proof of Theorem 1.3 : Scale invariance approach}

We first prove the following lemma:

Lemma 2.1. Let $\phi \in C^{1}(\mathbb{R})$ and $x=\phi(|y|) \frac{y}{|y|}$. Then, the following must hold

$$
\begin{gathered}
\frac{\partial x_{i}}{\partial y_{j}}=\frac{|x|}{|y|}\left[\delta_{i j}+\left(\frac{|y|}{|x|} \phi^{\prime}(|y|)-1\right) \frac{y_{i} y_{j}}{|y|^{2}}\right], \\
\operatorname{det}\left(\frac{\partial x}{\partial y}\right)=\frac{|y|}{|x|} \phi^{\prime}(|y|) \frac{|x|^{n}}{|y|^{n}}
\end{gathered}
$$

and

$$
\left|\nabla_{y} u(x)\right|^{2}=\left|\frac{\partial x}{\partial y} \nabla_{x} u(x)\right|^{2}=\frac{|x|^{2}}{|y|^{2}}\left(\left|\nabla_{\mathbb{S}^{n-1}} u(x)\right|^{2}+\left(\frac{|y|}{|x|} \phi^{\prime}(|y|)\right)^{2}\left|\nabla_{r} u(x)\right|^{2}\right) .
$$

Proof of Lemma 2.1. The equality (2.2) can be obtained by direct computations. Let $a:=\frac{|y|}{|x|} \phi^{\prime}(|y|)-1$. By (2.2), the Jacobian is given by

$$
\operatorname{det}\left(\frac{\partial x}{\partial y}\right)=\frac{|x|^{n}}{|y|^{n}} \operatorname{det}\left(\begin{array}{cccc}
a \frac{y_{1}^{2}}{|y|^{2}}+1 & a \frac{y_{1} y_{2}}{|y|^{2}} & \cdots & a \frac{y_{1} y_{n}}{|y|^{2}} \\
a \frac{y_{2} y_{1}}{|y|^{2}} & a \frac{y_{2}^{2}}{|y|^{2}}+1 & \cdots & \vdots \\
\vdots & \vdots & \ddots & \vdots \\
a \frac{y_{n} y_{1}}{|y|^{2}} & \cdots & \cdots & a \frac{y_{n}^{2}}{|y|^{2}}+1
\end{array}\right) \text {. }
$$


By the standard argument, one can see that 1 and $(a+1)$ are eigenvalues of the matrix, and their multiplicities are $n-1$ and 1 , respectively. Therefore,

$$
\operatorname{det}\left(\begin{array}{cccc}
a \frac{y_{1}^{2}}{|y|^{2}}+1 & a \frac{y_{1} y_{2}}{|y|^{2}} & \cdots & a \frac{y_{1} y_{n}}{|y|^{2}} \\
a \frac{y_{2} y_{1}}{|y|^{2}} & a \frac{y_{2}^{2}}{|y|^{2}}+1 & \cdots & \vdots \\
\vdots & \vdots & \ddots & \vdots \\
a \frac{y_{n} y_{1}}{|y|^{2}} & \cdots & \cdots & a \frac{y_{n}^{2}}{|y|^{2}}+1
\end{array}\right)=(a+1)=\frac{|y|}{|x|} \phi^{\prime}(|y|) .
$$

This proves (2.3). Now we need to prove (2.4). Since

$$
\frac{\partial x}{\partial y} \nabla u(x)=\left(\sum_{j=1} \frac{\partial x_{i}}{\partial y_{j}} \partial_{x_{j}} u\right)_{i=1, \ldots, n}=\frac{|x|}{|y|}\left(\nabla u+a \frac{x}{|x|} \nabla_{r} u\right),
$$

we have

$$
\begin{aligned}
\left|\frac{\partial x}{\partial y} \nabla u(x)\right|^{2} & =\frac{|x|^{2}}{|y|^{2}}\left(|\nabla u|^{2}+2 a\left|\nabla_{r} u\right|^{2}+a^{2}\left|\nabla_{r} u\right|^{2}\right) \\
& =\frac{|x|^{2}}{|y|^{2}}\left(\left|\nabla_{\mathbb{S}^{n-1}} u\right|^{2}+(a+1)^{2}\left|\nabla_{r} u\right|^{2}\right) .
\end{aligned}
$$

This yields (2.4) and completes the proof of Lemma 2.1,

Proof of Theorem 1.3(The inequality). We start the proof from the classical Caffarelli-Kohn-Nirenberg inequality. Let $1<p<n, \theta>1,0 \leq \frac{1}{\theta}-\frac{1}{\sigma} \leq \frac{1}{n}$ and $u \in C_{0}^{\infty}\left(B_{R}\right)$. Recall that the rescaled function $u_{\lambda}$ defined in (1.7) belongs to $C_{0}^{\infty}\left(B_{R}\right)$. Then we have

$$
S\left(\int_{B_{R}}|x|^{\frac{n \sigma}{p^{*}}}\left|u_{\lambda}(x)\right|^{\sigma} \frac{d x}{|x|^{n}}\right)^{\frac{1}{\sigma}} \leq\left(\int_{B_{R}}|x|^{\frac{n \theta}{p}}\left|\nabla u_{\lambda}(x)\right|^{\theta} \frac{d x}{|x|^{n}}\right)^{\frac{1}{\theta}} .
$$

We calculate both the sides of $(2.5)$ by considering the change of variables

$$
\begin{aligned}
z=x_{\lambda} & =\left[\lambda|x|^{-\frac{n-p}{p} \frac{\theta}{\theta-1}}+(1-\lambda) R^{-\frac{n-p}{p} \frac{\theta}{\theta-1}}\right]^{-\frac{p}{n-p} \frac{\theta-1}{\theta}} \frac{x}{|x|} \\
& =R \exp _{q}\left[\lambda \log _{q} \frac{|x|}{R}\right] \frac{x}{|x|} .
\end{aligned}
$$

Let $\phi(r)=R \exp _{q}\left[\lambda \log _{q} \frac{r}{R}\right]$. Then,

$$
\phi^{\prime}(r)=R\left(\exp _{q}\left[\lambda \log _{q} \frac{r}{R}\right]\right)^{q} \lambda\left(\frac{r}{R}\right)^{-q} \frac{1}{R}=\lambda\left(\frac{|z|}{|x|}\right)^{q} .
$$

Hence, we have

$\frac{|x|}{|z|} \phi^{\prime}(|x|)=\lambda\left(\frac{|x|}{|z|}\right)^{1-q}=1-\left(\frac{R}{|z|}\right)^{1-q}+\lambda\left(\frac{R}{|z|}\right)^{1-q}=(q-1) \log _{q} \frac{R}{|z|}+\lambda\left(\frac{R}{|z|}\right)^{1-q}$ 
and

$$
|x|^{\frac{n-p}{p}} \lambda^{-\frac{\theta-1}{\theta}}=|z|^{\frac{n-p}{p}}\left[(q-1) \log _{q} \frac{R}{|z|}+\lambda\left(\frac{R}{|z|}\right)^{1-q}\right]^{-\frac{\theta-1}{\theta}} .
$$

From (2.6), (2.7), and Lemma 2.1, we have

$$
\begin{aligned}
\int_{B_{R}}|x|^{\frac{n \sigma}{p^{*}}}\left|u_{\lambda}(x)\right|^{\sigma} \frac{d x}{|x|^{n}} & =\int_{B_{R}}|x|^{\frac{n-p}{p} \sigma-n} \lambda^{-\frac{\theta-1}{\theta} \sigma}|u(z)|^{\sigma}\left|\operatorname{det}\left(\frac{\partial x}{\partial z}\right)\right| d z \\
& =\int_{B_{R}}|z|^{\frac{n}{p^{*}} \sigma-n} \frac{|u(z)|^{\sigma}}{\left[\lambda\left(\frac{|z|}{R}\right)^{q-1}+(q-1) \log _{q} \frac{R}{|z|}\right]^{1+\frac{\theta-1}{\theta} \sigma} d z}
\end{aligned}
$$

The monotone convergence theorem shows us that the right hand side of (2.8) converges to the right hand side of Theorem 1.3 as $\lambda \rightarrow 0$. Similarly, Lemma 2.1 along with (2.6) and (2.7) implies that

$$
\begin{aligned}
\int_{B_{R}}|x|^{\frac{n \theta}{p}}\left|\nabla u_{\lambda}(x)\right|^{\theta} \frac{d x}{|x|^{n}} & \\
= & \int_{B_{R}}|x|^{\frac{n \theta}{p}} \lambda^{-\frac{\theta-1}{\theta} \theta}\left|\left(\frac{\partial z}{\partial x} \nabla u\right)(z)\right|^{\theta}\left|\operatorname{det}\left(\frac{\partial x}{\partial z}\right)\right| \frac{d z}{|x|^{n}} \\
& =\int_{B_{R}}|z|^{\frac{n}{p} \theta} \frac{\left[\left|\nabla_{\mathbb{S}^{n-1}} u\right|^{2}+\left(\lambda\left(\frac{|z|}{R}\right)^{q-1}+(q-1) \log _{q} \frac{R}{|z|}\right)^{2}\left|\nabla_{r} u\right|^{2}\right]}{\left[\lambda\left(\frac{|z|}{R}\right)^{\frac{\theta}{2}}+(q-1) \log _{q} \frac{R}{|z|}\right]^{\theta}} \frac{d z}{|z|^{n}} .
\end{aligned}
$$

The dominated convergence theorem as $\lambda \rightarrow 0$ yields the desired term in (1.19).

The homogeneous inequality (1.20) for $\theta=\sigma$ can be obtained by starting the argument from (1.16) instead of from (1.15). Indeed, the equality

$$
\int_{B_{R}}|x|^{\frac{n \theta}{p}}\left|\nabla_{r} u_{\lambda}(x)\right|^{\theta} \frac{d x}{|x|^{n}}=\int_{B_{R}}|x|^{\frac{n \theta}{p}}\left|\nabla_{r} u(x)\right|^{\theta} \frac{d x}{|x|^{n}}
$$

and (2.8) yield the homogeneous inequality (1.20) by taking $\lambda \rightarrow 0$. This completes the proof of the inequalities (1.19) and (1.20).

It now remains to be proven that (1.19) and (1.20) are scale invariant under the scaling (1.21). This can be done by direct calculation; however, we prove it in the next subsection by focusing the equivalence between classical inequalities (1.15)(1.16) and Theorem 1.3. We prove this at the end of Section 2.3.

\subsection{Proof of Theorem 1.3 : Transformation}

The following equivalence gives Theorem 1.3 . 
Proposition 2.1. Let $p<n, \theta>1,0 \leq \frac{1}{\theta}-\frac{1}{\sigma} \leq \frac{1}{n}, \frac{1}{p^{*}}=\frac{1}{p}-\frac{1}{n}$ and $q=1+\frac{n-p}{p} \frac{\theta}{\theta-1}$.

Then the following equalities

$$
\begin{aligned}
\int_{\mathbb{R}^{n}}|y|^{\frac{n \sigma}{p^{*}}}|v(y)|^{\sigma} \frac{d y}{|y|^{n}} & =\int_{B_{R}} \frac{|x|^{\frac{n \sigma}{p^{*}}}|u(x)|^{\sigma}}{\left[(q-1) \log _{q} \frac{R}{|x|}\right]^{1+\frac{\theta-1}{\theta} \sigma} \frac{d x}{|x|^{n}}} \\
\int_{\mathbb{R}^{n}}|y|^{\frac{n \theta}{p}}|\nabla v(y)|^{\theta} \frac{d y}{|y|^{n}} & =\int_{B_{R}}|x|^{\frac{n \theta}{p}}\left|L_{p, \theta} u(x)\right|^{\theta} \frac{d x}{|x|^{n}}, \\
\int_{\mathbb{R}^{n}}|y|^{\frac{n \theta}{p}}\left|\nabla_{r} v(y)\right|^{\theta} \frac{d y}{|y|^{n}} & =\int_{B_{R}}|x|^{\frac{n \theta}{p}}\left|\nabla_{r} u(x)\right|^{\theta} \frac{d x}{|x|^{n}}
\end{aligned}
$$

hold under the transformation

$$
u(x)=v(y), \quad y=R\left(-(q-1) \log _{q} \frac{|x|}{R}\right)^{-\frac{1}{q-1}} \frac{x}{|x|} .
$$

Proof of Proposition 2.1. For parameters $\alpha>0$ and $q>1$, we consider the transformation

$$
u(x)=v(y), \quad y=C\left(-\log _{q} \frac{|x|}{R}\right)^{-\alpha} \frac{x}{|x|}
$$

or equivalently

$$
x=R \exp _{q}\left[-\left(\frac{|y|}{C}\right)^{-\frac{1}{\alpha}}\right] \frac{y}{|y|}
$$

and choose suitable $\alpha, q$, and $C$ later. It follows from

$$
-\log _{q} \frac{|x|}{R}=-\frac{\left(\frac{|x|}{R}\right)^{1-q}-1}{1-q}=\left(\frac{|x|}{R}\right)^{1-q} \frac{\left(\frac{R}{|x|}\right)^{1-q}-1}{1-q}=\left(\frac{|x|}{R}\right)^{1-q}\left(\log _{q} \frac{R}{|x|}\right)
$$

that

$$
y=C\left(-\log _{q} \frac{|x|}{R}\right)^{-\alpha} \frac{x}{|x|}=C\left(\frac{|x|}{R}\right)^{\alpha(q-1)}\left(\log _{q} \frac{R}{|x|}\right)^{-\alpha} \frac{x}{|x|} .
$$

Let $\phi(r)=R \exp _{q}\left[-\left(\frac{r}{C}\right)^{-\frac{1}{\alpha}}\right]$. Direct computations with (2.10) give us the following:

$$
\begin{aligned}
\frac{|y|}{|x|} \phi^{\prime}(|y|) & =\frac{|y|}{|x|} R\left(\exp _{q}\left[-\left(\frac{|y|}{C}\right)^{-\frac{1}{\alpha}}\right]\right)^{q} \frac{1}{\alpha C}\left(\frac{|y|}{C}\right)^{-\frac{1}{\alpha}-1} \\
& =\frac{1}{\alpha}\left(\frac{|x|}{R}\right)^{q-1}\left(\frac{|y|}{C}\right)^{-\frac{1}{\alpha}}=\frac{1}{\alpha} \log _{q} \frac{R}{|x|}
\end{aligned}
$$


Now, we are ready to prove (2.14). By applying Lemma 2.1 with the help of (2.10) and (2.11), we have

$$
\begin{aligned}
\int_{\mathbb{R}^{n}}|y|^{\frac{n \theta}{p}}|\nabla v(y)|^{\theta} \frac{d y}{|y|^{n}} & \int_{B_{R}}|y|^{\frac{n \theta}{p}}-n\left|\frac{\partial x}{\partial y} \nabla u(x)\right|^{\theta}\left|\operatorname{det}\left(\frac{\partial y}{\partial x}\right)\right| d x \\
= & \int_{B_{R}}|y|^{\frac{n \theta}{p}}-n \frac{|x|^{\theta}}{|y|^{\theta}}\left(\left|\nabla_{\mathbb{S}^{n-1}} u\right|^{2}+\left(\frac{1}{\alpha} \log _{q} \frac{R}{|x|}\right)^{2}\left|\nabla_{r} u\right|^{2}\right)^{\frac{\theta}{2}} \frac{|y|^{n}}{|x|^{n}} \frac{\alpha}{\log _{q} \frac{R}{|x|}} d x \\
= & \alpha^{-\alpha \theta \frac{n-p}{p}} R^{-\alpha(q-1) \frac{n-p}{p} \theta} C^{\frac{n-p}{p} \theta} \\
& \times \int_{B_{R}} \frac{|x|^{\alpha(q-1) \frac{n-p}{p} \theta+\theta}\left(\left|\nabla_{\mathbb{S}^{n-1}} u(x)\right|^{2}+\left[\frac{1}{\alpha} \log _{q} \frac{R}{|x|}\right]^{2}\left|\nabla_{r} u(x)\right|^{2}\right)^{\frac{\theta}{2}}}{\left(\frac{1}{\alpha} \log _{q} \frac{R}{|x|}\right)^{\alpha \theta \frac{n-p}{p}+1}} \frac{d x}{|x|^{n}} .
\end{aligned}
$$

Here we take

$$
\alpha=\frac{p}{n-p} \frac{\theta-1}{\theta}, \quad q=1+\frac{n-p}{p} \frac{\theta}{\theta-1}, \quad C=R \alpha^{\alpha}
$$

so that

$$
\begin{array}{ll}
\alpha(q-1) \theta \frac{n-p}{p}+\theta=\frac{n \theta}{p}, & \alpha \theta \frac{n-p}{p}+1=\theta, \\
\alpha^{-\alpha \theta \frac{n-p}{p}} R^{-\alpha(q-1) \frac{n-p}{p} \theta} C^{\frac{n-p}{p} \theta}=1, & \frac{1}{\alpha}=q-1 .
\end{array}
$$

Therefore, the last term in (2.12) coincides with $\int_{B_{R}}|x|^{\frac{n \theta}{p}}\left|L_{p, \theta} u(x)\right|^{\theta} \frac{d x}{|x|^{n}}$. Applying a similar argument, it follows that

$$
\int_{\mathbb{R}^{n}}|y|^{\frac{n \theta}{p}}\left|\nabla_{r} v(y)\right|^{\theta} \frac{d y}{|y|^{n}}=\int_{B_{R}}|x|^{\frac{n \theta}{p}}\left|\nabla_{r} u(x)\right|^{\theta} \frac{d x}{|x|^{n}} .
$$

Finally, (2.3), (2.11), and (2.10) imply that

$$
\begin{aligned}
\int_{\mathbb{R}^{n}}|y|^{\frac{n \sigma}{p^{*}}}|v(y)|^{\sigma} \frac{d y}{|y|^{n}} & =\int_{B_{R}}|y|^{\frac{n \sigma}{p^{*}}-n}|u(x)|^{\sigma}\left|\operatorname{det}\left(\frac{\partial y}{\partial x}\right)\right| d x \\
& =\int_{B_{R}}|y|^{\frac{n \sigma}{p^{*}}-n}|u(x)|^{\sigma} \frac{|y|^{n}}{|x|^{n}} \frac{\alpha}{\log _{q} \frac{R}{|x|}} d x \\
& =\alpha^{-\frac{\theta-1}{\theta} \sigma} R^{-\frac{n-p}{p} \sigma} C^{\frac{n-p}{p} \sigma} \int_{B_{R}} \frac{|x|^{\frac{n \sigma}{p^{*}}}|u(x)|^{\sigma}}{\left[\frac{1}{\alpha} \log _{q} \frac{R}{|x|}\right]^{1+\frac{\theta-1}{\theta} \sigma} \frac{d x}{|x|^{n}}} .
\end{aligned}
$$

Applying (2.13), we have $\alpha^{-\frac{\theta-1}{\theta} \sigma} R^{-\frac{n-p}{p} \sigma} C^{\frac{n-p}{p} \sigma}=1$ and $\frac{1}{\alpha}=q-1$. This proves Proposition 2.1. 
As a consequence of Proposition 2.1, we obtain the inequalities (1.19) and (1.20) from the classical inequalities (1.15) and (1.16), respectively. Moreover, the function of the Aubin-Talenti type (1.8) in Theorems 1.1$] 1.2$ is obtained from the original Aubin-Talenti function (1.2) in $\mathbb{R}^{n}$ via the transformation.

Finally, we give a simple proof of the scale invariance of the inequalities (1.19)(1.20) by focusing the scaling structure between the dilation (1.3) and the scaling (1.21). Let $u \in C_{0}^{\infty}\left(B_{R}\right), v \in C_{0}^{\infty}\left(\mathbb{R}^{n}\right), \alpha>0, q>1$, and $\lambda>0$. We define the following transformations:

$$
\begin{array}{ll}
D_{\lambda}: C_{0}^{\infty}\left(\mathbb{R}^{n}\right) \rightarrow C_{0}^{\infty}\left(\mathbb{R}^{n}\right) ; \quad D_{\lambda} v(y):=v(\lambda y), \\
S_{\lambda}: C_{0}^{\infty}\left(B_{R}\right) \rightarrow C_{0}^{\infty}\left(B_{R}\right) ; \quad S_{\lambda} u(x):=u\left(x_{\lambda}\right), \quad x_{\lambda}:=R \exp _{q}\left[\lambda \log _{q} \frac{|x|}{R}\right] \frac{x}{|x|} \\
T_{\alpha}: C_{0}^{\infty}\left(\mathbb{R}^{n}\right) \rightarrow C_{0}^{\infty}\left(B_{R}\right) ; \quad T_{\alpha, q} v(x):=v(y), \quad y:=R\left(-(q-1) \log _{q} \frac{|x|}{R}\right)^{-\alpha} \frac{x}{|x|}
\end{array}
$$

and its inverse transformation $T_{\alpha, q}^{-1}$.

Theorem 2.1. For all $\lambda, \alpha>0, q>1, u \in C_{0}^{\infty}\left(B_{R}\right)$, and $v \in C_{0}^{\infty}\left(\mathbb{R}^{n}\right)$, there hold

$$
\begin{aligned}
& S_{\lambda}\left(T_{\alpha, q} v\right)(x)=T_{\alpha}\left(D_{\lambda^{-\alpha}} v\right)(x) \quad \text { for all } x \in B_{R}, \\
& T_{\alpha}^{-1}\left(S_{\lambda} u\right)(y)=D_{\lambda^{-\alpha}}\left(T_{\alpha, q}^{-1} u\right)(y) \quad \text { for all } y \in \mathbb{R}^{n} .
\end{aligned}
$$

Proof of Theorem 2.1. Fix $v \in C_{0}^{\infty}\left(\mathbb{R}^{n}\right)$. Then,

$$
\begin{aligned}
S_{\lambda}\left(T_{\alpha, q} v\right)(x) & =v\left(\left(-\lambda(q-1) \log _{q} \frac{|x|}{R}\right)^{-\alpha}\right) \\
& =\left(D_{\lambda^{-\alpha}} v\right)\left(\left(-(q-1) \log _{q} \frac{|x|}{R}\right)^{-\alpha}\right)=T_{\alpha, q}\left(D_{\lambda^{-\alpha}} v\right)(x)
\end{aligned}
$$

for all $x \in B_{R}$. The other equality is obtained by taking $v=T_{\alpha, q}^{-1} u$.

Applying Theorem 2.1 and Proposition 2.1 with the help of the dilation invariance of the classical inequalities (1.15) and (1.16), we prove the scale invariance properties of (1.19) and (1.20) under the scaling (1.21).

Proof of Theorem 1.3 (Scale invariance). Let us define some notations for norms in Theorem 1.3, which simplify the proof of the scale invariance.

$$
\begin{array}{ll}
\|v\|_{X\left(\mathbb{R}^{n}\right)}:=\left(\int_{\mathbb{R}^{n}}|y|^{\frac{n \sigma}{p^{*}}}|v(y)|^{\sigma} \frac{d y}{|y|^{n}}\right)^{\frac{1}{\sigma}}, & \|v\|_{Y\left(\mathbb{R}^{n}\right)}:=\left(\int_{\mathbb{R}^{n}}|y|^{\frac{n \theta}{p}}|v(y)|^{\theta} \frac{d y}{|y|^{n}}\right)^{\frac{1}{\theta}}, \\
\|u\|_{\tilde{X}\left(B_{R}\right)}:=\left(\int_{B_{R}} \frac{|x|^{\frac{n \sigma}{p^{*}}}|u(x)|^{\sigma}}{\left.\left[(q-1) \log _{q} \frac{R}{|x|}\right]^{1+\frac{\theta-1}{\theta} \sigma} \frac{d x}{|x|^{n}}\right)^{\frac{1}{\sigma}}, \quad\|u\|_{\tilde{Y}\left(B_{R}\right)}:=\left(\int_{B_{R}}|x|^{\frac{n \theta}{p}}|u(x)|^{\theta} \frac{d x}{|x|^{n}}\right)^{\frac{1}{\theta}} .}\right.
\end{array}
$$


Assume that $\alpha, q$ satisfy (2.13). Then, Proposition 2.1 can be stated as

$$
\left\|T_{\alpha, q}^{-1} u\right\|_{X\left(\mathbb{R}^{n}\right)}=\|u\|_{\tilde{X}\left(B_{R}\right)} \quad \text { and } \quad\left\|\nabla\left(T_{\alpha, q}^{-1} u\right)\right\|_{Y\left(\mathbb{R}^{n}\right)}=\left\|L_{p, \theta} u\right\|_{\tilde{Y}\left(B_{R}\right)} .
$$

Furthermore, the scaling (1.21) coincides with $u_{\lambda}(x)=\lambda^{-\frac{\theta-1}{\theta}}\left(S_{\lambda} u\right)(x)$.

Using these notation, we prove the invariance of $\|u\|_{\tilde{X}\left(B_{R}\right)}$ and $\left\|L_{p, \theta} u\right\|_{\tilde{Y}\left(B_{R}\right)}$ under the scaling $\lambda^{-\frac{\theta-1}{\theta}} S_{\lambda}$. It follows from (2.15) that

$$
\begin{aligned}
& \left\|\lambda^{-\frac{\theta-1}{\theta}} S_{\lambda} u\right\|_{\tilde{X}\left(B_{R}\right)}=\left\|\lambda^{-\frac{\theta-1}{\theta}} T_{\alpha, q}^{-1}\left(S_{\lambda} u\right)\right\|_{X\left(\mathbb{R}^{n}\right)}, \\
& \left\|\lambda^{-\frac{\theta-1}{\theta}} L_{p, \theta}\left(S_{\lambda} u\right)\right\|_{\tilde{Y}\left(B_{R}\right)}=\left\|\lambda^{-\frac{\theta-1}{\theta}} \nabla\left(T_{\alpha, q}^{-1}\left(S_{\lambda} u\right)\right)\right\|_{Y\left(\mathbb{R}^{n}\right)} .
\end{aligned}
$$

Applying Theorem 2.1 and the dilation invariance in $\mathbb{R}^{n}$ with $\lambda^{-\frac{\theta-1}{\theta}}=\left(\lambda^{-\alpha}\right)^{\frac{n-p}{p}}$, we obtain

$$
\begin{aligned}
& \left\|\lambda^{-\frac{\theta-1}{\theta}} T_{\alpha, q}^{-1}\left(S_{\lambda} u\right)\right\|_{X\left(\mathbb{R}^{n}\right)}=\left\|\lambda^{-\frac{\theta-1}{\theta}} D_{\lambda^{-\alpha}}\left(T_{\alpha, q}^{-1} u\right)\right\|_{X\left(\mathbb{R}^{n}\right)}=\left\|T_{\alpha, q}^{-1} u\right\|_{X\left(\mathbb{R}^{n}\right)} \\
& \left\|\lambda^{-\frac{\theta-1}{\theta}} \nabla\left(T_{\alpha, q}^{-1}\left(S_{\lambda} u\right)\right)\right\|_{Y\left(\mathbb{R}^{n}\right)}=\| \lambda^{-\frac{\theta-1}{\theta}} \nabla\left(D_{\lambda^{-\alpha}}\left(T_{\alpha, q}^{-1} u\right)\left\|_{Y\left(\mathbb{R}^{n}\right)}=\right\| \nabla\left(T_{\alpha, q}^{-1} u\right) \|_{Y\left(\mathbb{R}^{n}\right)} .\right.
\end{aligned}
$$

Then, the desired invariance holds by applying (2.15) to the last equalities.

Acknowledgments. This work was partially funded by JSPS KAKENHI \# 18K13441.

\section{References}

[1] Adimurthi, do Ó, J. M., Tintarev, K., Cocompactness and minimizers for inequalities of Hardy-Sobolev type involving $N$-Laplacian, NoDEA Nonlinear Differential Equations Appl. 17 (2010), 467-477.

[2] Alvino, A., Sulla diseguaglianza di Sobolev in spazi di Lorentz, Boll. Un. Mat. Ital. A (5) 14 (1977), 148-156.

[3] Aubin, T., Problèmes isopérimétriques et espaces de Sobolev, J. Differ. Geom. 11 (1976), 573-598.

[4] Caffarelli, L., Kohn, R., Nirenberg, L., First order interpolation inequalities with weights, Compositio Math. 53 (1984), 259-275.

[5] Cassani, D., Ruf, B., Tarsi, C., Optimal Sobolev Type Inequalities in Lorentz Spaces, Potential Anal. 39 (2013), 265-285.

[6] Cassani, D., Sani, F., Tarsi, C., Equivalent Moser type inequalities in $\mathbb{R}^{2}$ and the zero mass case, J. Funct. Anal. 267 (2014), 4236-4263.

[7] Cianchi, A., A sharp embedding theorem for Orlicz-Sobolev spaces, Indiana Univ. Math. J. 45 (1996), 39-65. 
[8] Costa, D., Tintalev, C., Concentration profiles for the Trudinger-Moser functional are shaped like toy pyramids, J. Funct. Anal. 266 (2014), 676-692.

[9] Davies, E. B., A review of Hardy inequalities, Oper. Theory Adv. Appl. 110, Birkhäuser, Basel, 1999, 55-67.

[10] Federer, H., Fleming, W., Normal and integral currents, Ann. of Math. (2) 72 (1960), 458-520.

[11] Horiuchi, T., Kumlin, P., On the Caffarelli-Kohn-Nirenberg type inequalities involving critical and supercritical weights, Kyoto J. Math. 52 (2012), 661-742.

[12] Ioku, N., Ishiwata, M., A Scale Invariant Form of a Critical Hardy Inequality, Int. Math. Res. Not. 18 (2015), 8830-8846.

[13] Ioku, N., Ishiwata, M., A Note on the Scale Invariant Structure of Critical Hardy Inequalities, Geometric Properties for Parabolic and Elliptic PDE's, Springer Proceedings in Mathematics \& Statistics 176 (2016), 97-120.

[14] Kesavan, S., Symmetrization \& applications, Series in Analysis, 3. World Scientific Publishing Co. Pte. Ltd., Hackensack, NJ, 2006.

[15] Opic, B., Kufner, A., Hardy-type inequalities, Pitman Research Notes in Mathematics Series 219, Longman Scientific \& Technical, Harlow, 1990.

[16] Maz'ya, V.G., Classes of domains and imbedding theorems for function spaces, Soviet Math. Dokl. 1 (1960), 882-885.

[17] Sano, M., Takahashi, F., Scale invariance structures of the critical and the subcritical Hardy inequalities and their improvements, Calc. Var. Partial Differential Equations 56 (2017), no. 3, Art. 69, 14 pp.

[18] Suyari, H., Fundamental Mathematics for Complex Systems, Makino shoten, Tokyo, 2010.

[19] Takahashi, F., A simple proof of Hardy's inequality in a limiting case, Arch. Math. 104 (2015), 77-82.

[20] Talenti, G., Best constant in Sobolev inequality, Ann. Mat. Pura Appl. 110 (1976), 353-372.

[21] Tsallis, C., Possible generalization of Boltzmann-Gibbs statistics, J. Statist. Phys. 52 (1988), 479-487.

[22] Tsallis, C., Introduction to Nonextensive Statistical Mechanics: Approaching a Complex World, Springer, NewYork, 2009.

[23] Willem, M., Minimax theorems, Progress in Nonlinear Differential Equations and their Applications, 24. Birkhäuser Boston, Inc., Boston, 1996. 